\title{
Application design of piezoelectric effect in cabin seat belt status indication
}

\author{
Lixia Gao, Hanlin Shen \\ Aviation Engineering Institute, Civil Aviation Flight University of China, Guanghan City, Sichuan Province, China
}

\begin{abstract}
During the special operation stage of the aircraft, if the passengers did not fasten their safety belts in accordance with the requirements, it may bring unexpected injury, resulting in unnecessary losses. So if there is a corresponding system can help the crew to accurately confirm the state of the safety belts of the passengers under special circumstances, what we said before can be avoided. The seat belt status indicator system is designed based on the piezoelectric effect can help the crew to know the state of the safety belt clearly, to ensure the effectiveness of their work.
\end{abstract}

\section{Introduction}

Because of the particularity of the operation of the aircraft, especially during the stage of take-off, landing and air turbulence encountered, the safety belt protection to passengers is particularly important. Under the existing circumstances, the plane crew need to repeat one by one in order to confirm repeatedly and remind constantly the passengers to use seat belts. Under some special circumstances, some of the passengers do not listen to the crew in time, so it easily leads to accidental injury. If the crew can make sure of the actual state of the safety belts of all passengers in the crew work area, it can reduce the workload of the crew, help the crew to give a timely reminder, ensure the effectiveness of the crew work, guarantee the safety of passengers.

\section{Design concept}

Piezoelectric material is a kind of material which is sensitive to machine, electricity, sound, light and heat. It is widely used in the field of the sensor, transducer, nondestructive testing and communication technology. The pressure can be caused by the action of the seat belt buckle. The piezoelectric material is installed in the corresponding position to accurately identify the safety belt buckle or not. The piezoelectric material is using to feel the pressure, and converts the pressure signal into corresponding electrical signals. The safety belt state display device is set in the service area. The induction electrical signal from the piezoelectric material will be sent to the corresponding display device. The crew can accurately identify with the safety state of all the passengers. A timely reminder will be given to the corresponding passengers.

The performance of piezoelectric materials is directly related to the accuracy of the acquisition signal. It will affect the correctness of the display. It can eventually determine whether the system is successful. The piezoelectric ceramic is a kind of important piezoelectric materials. It has excellent piezoelectric properties, so it is used in the application design.

\section{Design scheme}

The seat belt status indicator system is designed based on the piezoelectric effect. The hardware design includes power module, piezoelectric signal acquisition module, signal processing module, the main processor module and status indication module. Among them, the acquisition and processing of piezoelectric materials is the focus of the entire design. The basic hardware structure of system diagram as shown in figure 1 .



Figure 1. Basic hardware structure of the system

Considering the work environment characteristics and the demand of I/O, the TI MSP430 is chosen as the main processor. The power module can complete the conversion of electrical energy form. It supplies the power to the signal processing circuit, system voltage comparator, main processor and indication module. In this system, the main processor needs to be provided $3 \mathrm{~V}$ 
working voltage, the indicating module needs to provided $5 \mathrm{~V}$ voltage, the signal processing circuit and the comparison circuit need to be provided working voltage of $12 \mathrm{~V}$. Through a combination of considerations, an AC-DC step-down power module is chosen to meet the circuit power supply demand. It can provide a stable voltage source of $3 \mathrm{~V}, 5 \mathrm{~V}$ and $12 \mathrm{~V}$. The signal processing circuit finishes the signal processing from piezoelectric ceramics. The result is sent to the next level circuit for further processing. The main processor circuit completes collection the outputs form the comparison circuit and the collected signal is processed correspondingly. The processing result is sent to the corresponding indicating position to trigger the indicating system to work.

\section{Piezoelectric signal obtaining and processing}

\section{1. piezoelectric signal analysis}

With the different compression modes and different intensity of the situation, the piezoelectric ceramics generates the electric signals with different size and stability. In order to obtain the characteristics of electric signals generating under different conditions, and to complete the accurate judgment of the actual situation according to the characteristics, so to provide accurate signal to the processor to processor output to the indicator system clear signal, thus the performance test of piezoelectric ceramic of the selected type must be finished firstly. When the piezoelectric ceramic is triggered, the signal appeared in short time and each time the trigger is not the same. So in order to clarify the characteristics and laws of the signal, a large number of signals are tracking and acquisition by the oscilloscope. And the signal is statistically analyzed, to prepare for the subsequent design of the circuit.

According to the diameter of the selected piezoelectric sheet, specific test for different situations was done. Through a lot of tests and comparison, more clutter signal will be found out in the signal waveform when the pressure is far from the central zone of the piezoelectric plate. A waveform is generated by a lateral press as shown in Figure 2.

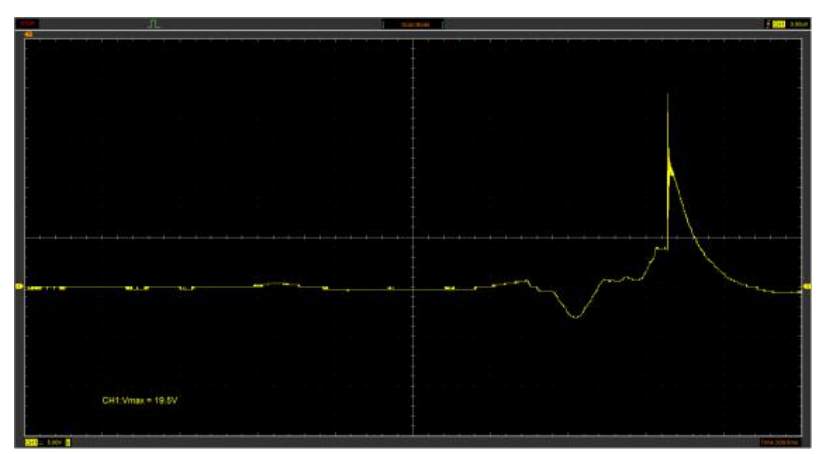

Figure 2. Waveform generated by a lateral press
If the pressure position is near the central zone of the piezoelectric plate, the clutter signal of the waveform is less. The waveform is complete and clear, convenient for further analysis, processing and use.

But if the pressure is different, there is a big difference between the voltage signals when the piezoelectric ceramic is pressed. One voltage waveform signal obtained by a light touch of the central zone of the piezoelectric plate is shown in Figure 3. The short-term peak voltage is $6.43 \mathrm{~V}$, and the voltage signal duration is short.

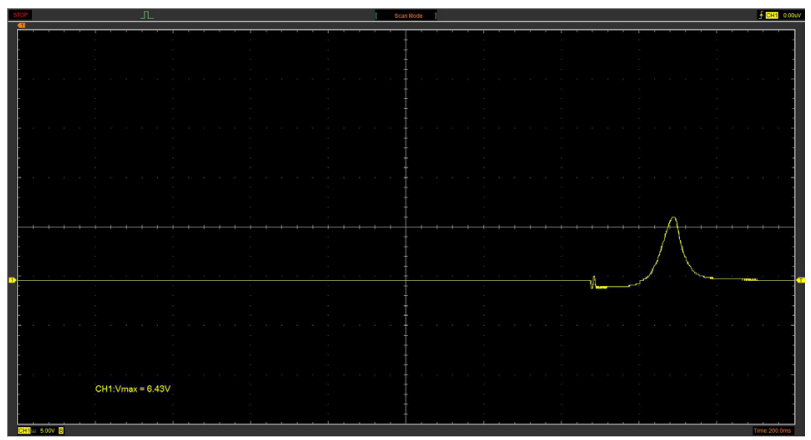

Figure 3. One voltage waveform from a light touch

One voltage waveform obtained by pressing the central zone of the piezoelectric plate is shown in Figure 4. The peak value of short-time voltage is $23.7 \mathrm{~V}$, and the voltage signal duration is longer.

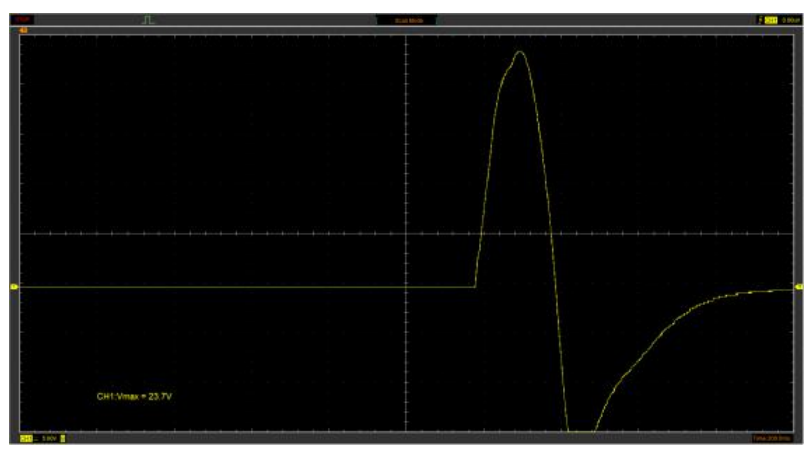

Figure 4. One voltage waveform from a normal touch

A large number output signals of the sensors are obtained by many tests of different forms and potency dimensions. Through the comparison of the output signals, it is found out that the short voltage value generated by the piezoelectric ceramic is more than $20 \mathrm{~V}$ when the piezoelectric ceramic is under enough pressure. If the pressing pressure is insufficient or touch case, the short voltage values are below $12 \mathrm{~V}$.

\subsection{Comparison circuit design}

The comparison circuit design is used to identify the height of the piezoelectric ceramic output voltage. According to the large number of measurement results and their distribution characteristics, the state of the 
corresponding device will be identified. It is triggered normally, or not triggered, or false triggered. It will help to avoid error indication.

Since the voltage range of the generated voltage is just confirmed to identify whether the device has been triggered or not, so the voltage comparator circuit is used to complete the corresponding function.

LM339 is the voltage comparator professional. It is a four differential comparator including four independent voltage comparators. The characteristics for it include fast switching speed, small delay time, a wide range of power supply voltage. The single power supply is $2 \sim$ $36 \mathrm{~V}$, and the double power supply voltage is $+1 \mathrm{~V} \sim+$ $18 \mathrm{~V}$. It can be used for special occasions. In addition, its ratio differential input voltage range is large enough to be equal to the supply voltage. And the output potential can be selected flexibly and conveniently. So it is suitable for the voltage comparison circuit. The circuit principle diagram of the comparison circuit is shown in Figure 5.

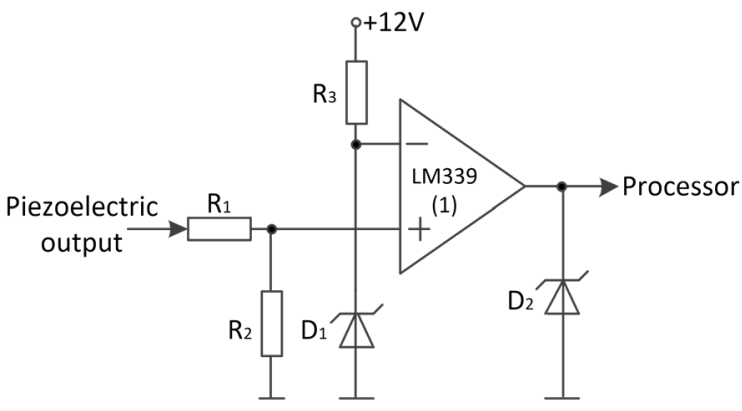

Figure 5. Comparison circuit principle diagram

According to the measuring result analysis, under the normal and abnormal pressure, the voltage value obtained is higher than $20 \mathrm{~V}$ and lower than $12 \mathrm{~V}$. The voltage scope distinction is clear. In order to ensure the normal pressure is identified and the abnormal pressure is filtered out, the circuit hardware parameters are reasonably designed. $\mathrm{R}_{1}, \mathrm{R}_{2}$ and $\mathrm{R}_{3}$ are respectively designed as $10 \mathrm{k} \Omega, 6 \mathrm{k} \Omega$ and $5 \mathrm{k} \Omega, \mathrm{D}_{1}$ as a $6 \mathrm{~V}$ regulator.

The comparative results for the voltage comparator will be sent to the microcontroller to identify. So the voltage values must meet the processor voltage input range. In order to meet the requirements of signal, a stabilivolt with the corresponding parameters is set in the circuit, before the microcontroller and after comparison circuit. When a high voltage signal comes from the comparator, it can be limited to a fixed value, and this value is no high than the requirement of the microcontroller. Thus, a $4.5 \mathrm{~V}$ regulator is used in the circuit after the output end of the comparator. When the comparator output is in high level, the signal sent to the main processor size does not exceed $4.5 \mathrm{~V}$, to meet the requirements of the size of the input signal processor.

\subsection{Eliminate false triggering design}

In order to reduce the possibility of the false triggering further to some extent, those voltage signals obtained by normal pressing, edge pressing and gent pressing are sent to the comparison circuit to deal with. Through a large number of tests, it is clear that the high level signal output can be stable for a long time. A certain measurement effect is shown in Figure 5.

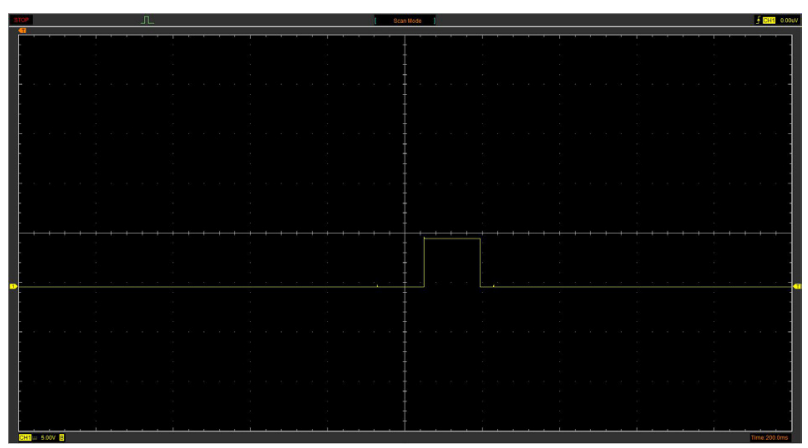

Figure 6. A certain measurement effect for normal pressure

In the case of false triggering, the comparator circuit even produces a high level output, and the output signal has a shorter duration, as shown in Figure 6.

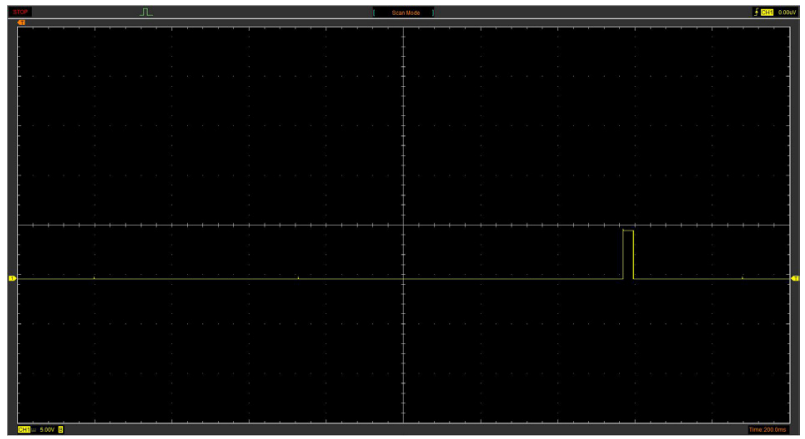

Figure 7. A certain measurement effect for false triggering

According to a large number of repeated test statistics, there is an obvious difference in duration between the normal pressing and abnormal pressing. The time of duration of the abnormal is about no more than $100 \mathrm{~ms}$. Therefore, to reduce the possibility of false triggering further, during the system programming, the high level signal delay time is obtained and compared, and the value which is less than $100 \mathrm{~ms}$ is believed to be from false triggering. So the error triggering signal is filtered further.

\section{Status indicating}

The display system of the design should be able to realize the explicit directions of each seat belt state. This function can be achieved by using LED lamp or LCD display. Initially, all seats corresponding state indicator lamp is lit. When the corresponding seat safety belts are 
buckled, the corresponding state indicator lamp is off. If the safety belt is released again, this kind of touch will make the indicator lamp light again. And the next safety belt buckle action will leads to the corresponding indicator lights go out again. The logic design is applied to the practical situation that the safety belt is buckled and unlocked many times during the operation of the aircraft.

\section{Conclusions}

This system has the advantages of simple structure, low cost, high reliability. And it has great practical value, not only can be used in the plane, but also can be used in high iron and some special entertainment facilities. The performance of the system is closely related to reliability of piezoelectric ceramics which is chosen, thus the performance improvement of the material will make the system stability and reliability to be further improved.

\section{References}

1. Xu Bin, Guo Xueyi, Chen Hongbing. Active Icing Monitoring for Wind Turbine Blade Models with PZT Technology. Piezoelectrics \& Acoustooptics, 39(2017).

2. Zhang Chuanjuan. Study of a compound piezoelectric energy harvester. Power Supply Technology and its Application, 19 (2015).

3. Yang Gaofeng. Preparation of Alarm Equipment PZT Piezoelectric Ceramics. Ceramics, 43( 2017)

4. Yuan Jiangbo. Power Generation and Pivotal Technique of Discal Energy Harvester (doctoral thesis), Harbin Institute of Technology (2011).

5. Congjian Li. Research on Energy Harvester Used in Roads Based on Piezoelectric Effect (master's thesis). Harbin Institute of Technology, (2015).

6. Xian Yongbiao, Li Ketian. Design of a Piezoelectric Ceramic Driver. Mechanical and Electrical Engineering Technology, 46(2017). 